\title{
Confining string beyond the free approximation: the case of random percolation
}

\section{Pietro Giudice*, Ferdinando Gliozzi, Stefano Lottini}

Dipartimento di Fisica Teorica, Università di Torino and INFN, sezione di Torino, Italy E-mail: giudicedto.infn.it, gliozzidto.infn.it, lottini@to.infn.it

\begin{abstract}
The random percolation model can be viewed as the dual of a well defined confining gauge theory; since this theory, having no Monte Carlo dynamics at all, is simple to simulate, it is possible to study the properties of the flux tube with very high precision; we show it can be described by the effective string picture. Our results are lattice regularisation independent, therefore they are well defined also in the continuum limit, and, for the first time in a gauge theory, it has been possible to determine the next-to-leading quantum corrections throughout the computation of the $T^{6}$ coefficient of the Taylor expansion of $\sigma(T)$. Furthermore, this coefficient results to be related to the universal ratio $T_{c} / \sqrt{\sigma_{0}}$.
\end{abstract}

The XXVI International Symposium on Lattice Field Theory

July 14 - 19, 2008

Williamsburg, Virginia, USA

\footnotetext{
* Speaker.
} 


\section{Introduction: the effective string theory}

The assumption behind the effective string theory is that the color flux connecting a pair of quark is squeezed inside a thin flux tube; as a consequence, the confining potential rises linearly. According to this picture the flux tube should behave as a free vibrating string.

Unfortunately, the action of this effective theory is unknown; the simplest assumpion is that it is described by the Nambu-Goto action, which is proportional to the string worldsheet area.

We try to summarize the outcome of many studies on this argument discussing some properties of the first terms of the low temperature expansion of the string tension (the first term $\sigma_{0}$ is the zerotemperature string tension):

$$
\sigma(T)=\sigma_{0}-(d-2) \frac{\pi}{6} T^{2}+\sum_{n \geq 3} c_{n} T^{n}
$$

The second term, the analogue of the Lüscher term at finite temperature which was calculated in Ref. [1], does not depend on the gauge group and is expected to be independent of the interaction terms of the effective theory. Thanks to a certain open-closed string duality it was shown that for any dimensionality $c_{3}=0$ and, in three dimensions, $c_{4}$ is universal [2]; hence, it coincides with the value calculated in the NG model [月, 那:

$$
c_{4}=-(d-2) \frac{\pi^{2}}{72 \sigma_{0}} .
$$

Using a different approach to the effective string theory, Ref. [5], the above results were confirmed for all values of $d$.

In this paper we will evaluate the coefficients $c_{n}$ up to $n=6$, in a simple, but not trivial, model: the gauge theory dual to the $3 d$ percolation model. All of the date agree with the universal values of $c_{2}$ and $c_{4}$ and lead to $c_{5}=0$ and $c_{6}=\pi^{3} /\left(C \sigma_{0}^{2}\right)$, where $C \simeq 300$.

We decided to focus our attention to the behaviour of the Polyakov-Polyakov correlation function $\left\langle P(0) P^{*}(r)\right\rangle$ at finite temperature $T=\frac{1}{a \ell}$ in $(2+1)$-dimensions; $r$ is the distance between the Polyakov loops, $\ell$ is the time extent of the lattice and $a$ is the lattice spacing.

The functional form of the correlator has been calculated at the next-to-leading order (NLO) in Ref. [П]:

$$
\left\langle P(0) P^{*}(r)\right\rangle_{N L O}=\frac{e^{-\mu \ell-\tilde{\sigma} r \ell}}{\eta(\tau)^{d-2}}\left(1-\frac{(d-2) \pi^{2} \ell\left[2 E_{4}(\tau)-E_{2}^{2}(\tau)\right]}{1152 \tilde{\sigma} r^{3}}+O\left(\frac{1}{r^{5}}\right)\right),
$$

where $\eta$ is the Dedekind function, $E_{4}$ and $E_{2}$ the two Eisenstein functions and $\tau=i \ell /(2 r)$.

Using Eq. (1.3) one can find, for asymptotically large $r$ :

$$
\sigma(T)=\tilde{\sigma}-\frac{\pi}{6} T^{2}-\frac{\pi^{2}}{72 \tilde{\sigma}} T^{4}=\sigma_{0}-\frac{\pi}{6} T^{2}-\frac{\pi^{2}}{72 \sigma_{0}} T^{4}+O\left(T^{5}\right) .
$$

Note here the difference between $\tilde{\sigma}$ and $\sigma_{0}: \tilde{\sigma}=\sigma_{0}+O\left(T^{5}\right)$. 


\section{The gauge theory dual to the percolation model}

In this paper we study a particular gauge theory, first introduced in Ref. [6], that is dual to the random percolation model. A more complete account will be presented in Ref. [0]].

It is known in three dimensions it is possible to study a well defined $S_{Q}$-gauge ${ }^{1}$ theory dual to the $Q$-state Potts model through the Kramers-Wannier duality [8]. Thanks to the fact one can map some gauge invariant observables, such as Wilson loops and Polyakov correlators, into the corresponding quantities of the spin model, it is numerically convenient to inspect the properties of the dual theory instead of those of the gauge model. The ingredient which is then fundamental in our approach is the Fortuin-Kasteleyn reformulation [9] of the $Q$-state Potts model, by which it is possible to determine gauge observables in a very efficient way. This approach, that for $Q>1$ is only a powerful numerical method, can be applied to the random percolation model whose gauge formulation is not known: it is the gauge theory in the $Q \rightarrow 1$ limit.

The key ingredient is the method used to calculate the Wilson loops in this setup: we define a procedure to determine its value studying some topological properties of the dual model.

The connected components of the graph, formed by active links, are known as clusters; $W_{\gamma}$ is the value of the Wilson loop associated with a loop with contour $\gamma$. We set $W_{\gamma}=1$ if there is no cluster topologically linked to the contour $\gamma$, otherwise we set $W_{\gamma}=0$. The same linking properties are used to determine the Polyakov-Polyakov loop correlators $\left\langle P(0) P^{*}(r)\right\rangle$ : at finite temperature the contour $\gamma$ is a $r \times \ell$ rectangle, with two sides identified.

Another interesting study of this model, related to the monopole mass, can be found in [10].

\section{Simulations}

The idea behind this work is not only to verify whether one can observe the presence of shape effect due to rough fluctuations of the string, in agreement with the universality predictions of the effective string picture (as a matter of fact we have discussed this point in Ref. [11]); we also would verify that our results are not regularisation dependent. In other words, we would discuss if our results describe a "real" phenomenon and not a lattice/model artifact.

We therefore study five different systems (see Table 1) characterized by different occupancy probability $p$, different kind of percolation (site or bond) and different geometry of the lattice (simple cubic lattice (SC) and body-centered cubic lattice (BCC)).

We worked on a lattice of size $L^{2} \times \ell$, where $\ell$, the inverse of the temperature, was chosen such that $0.3 T_{c} \lesssim T \lesssim 0.8 T_{c}$. The value of the spatial size was

\begin{tabular}{|c|c|c|}
\hline Lattice & $p$ & $\ell_{c}=1 / a T_{c}$ \\
\hline SC bond & 0.272380 & 6 \\
SC bond & 0.268459 & 7 \\
SC bond & 0.265615 & 8 \\
SC site & 0.3459514 & 7 \\
BCC bond & 0.21113018 & 3 \\
\hline
\end{tabular}

Table 1: The five systems simulated. $L=128$ which was in most cases sufficient to account for the infinite volume limit. Just in the case $\ell_{c}=8$, simulated at $\ell=10$ and $\ell=11$, we found a sizable dependence on the lattice size $L$; in this case we performed further simulations on larger lattices in order to extract the correct value of $\tilde{\sigma}$ using the scaling relation $(v=4 / 3$ is the termal exponent of $2 d$ percolation model):

$$
\tilde{\sigma}_{1 / L}=\tilde{\sigma}-c L^{-1 / v}
$$

\footnotetext{
${ }^{1} S_{Q}$ is the symmetric group.
} 
For each system, we measured $\left\langle P(0) P^{*}(r)\right\rangle$ by varying the distance between the two Polyakov lines from $r=8$ to $r=50$; to reach an acceptable statistics, we collected data from $10^{5}$ configurations for each value of $p$ and $\ell$.

The algorithm used, described in detail in Ref. [6], is basically aimed at determining the linking properties of clusters with the Polyakov-Polyakov contour.

\section{Numerical results}

Our numerical results are compared with the expected behaviour of the Polyakov-Polyakov correlation function given in Eq. (1.3). Being an expression valid in the infrared limit we use a sliding window analysis to determine the correct values of the fitted parameter $\tilde{\sigma}$ : we fitted the data in the range $r_{\min } \leq r \leq r_{\max }$ by progressively discarding the short distance data, varying $r_{\text {min }}$ but fixing the value of $r_{\max }=50 a$ (see Fig. 1). In all five sistems considered a large plateau appears for all values of $\ell$ not too close to $\ell_{c}$, showing the stability of the fits and so the suitability of the string picture to describe our data. It is important to note that, as Fig. 11 shows, there are different values of the string tension for different values of $\ell$, i.e. of $T$. In other words, the value of $\tilde{\sigma}$ is not yet the string tension at zero-temperature $\sigma_{0}$; the formula Eq. (1.3) is not the exact formula because it only takes into account the temperature dependence up to the order $T^{4}$ (see Eq. (1.4)). We studied the dependence of $\tilde{\sigma}$ on $\ell$ and we verified, in all cases, that for $a T=1 / \ell$ low enough the correction is proportional to $T^{6}$ (see Fig. 2). Therefore, we used the value of $\tilde{\sigma}$ to determine the value of $\sigma(T)$ by Eq. (1.4), i.e. we reconstructed the correct dependence of the string tension on the temperature; then we used these data to perform a new fit to determine the first model-dependent term by means of the Ansatz:

$$
\sigma(T)=\sigma_{0}-\frac{\pi}{6} T^{2}-\frac{\pi^{2}}{72 \sigma} T^{4}+\frac{\pi^{3}}{C \sigma_{0}^{2}} T^{6}+O\left(T^{8}\right) .
$$

Thereby, we can identify stable values both for the zero-temperature string tension $\sigma_{0}$ and the coefficient $C$, see Table 2 .

\begin{tabular}{|c|c|c|c|c|}
\hline Lattice & $\ell_{c}=1 / a T_{c}$ & $C$ & $a^{2} \sigma_{0}$ & $T_{c} / \sqrt{\sigma_{0}}$ \\
\hline SC bond & 6 & $291(7)$ & $0.012612(6)$ & $1.4841(4)$ \\
SC bond & 7 & $281(5)$ & $0.009234(5)$ & $1.4866(5)$ \\
SC bond & 8 & $297(5)$ & $0.007059(5)$ & $1.4878(5)$ \\
SC site & 7 & $307(9)$ & $0.009399(8)$ & $1.4735(6)$ \\
BCC bond & 3 & $295(14)$ & $0.0474(4)$ & $1.531(7)$ \\
\hline
\end{tabular}

Table 2: The parameters $C$ and $a^{2} \sigma_{0}$ in the fit (4.1) for the numerical experiments listed in Table 1. The last column is the universal ratio $T_{c} / \sqrt{\sigma_{0}}$ as obtained by combining the second and the fourth columns.

Note that the five values of $C$ coincide up to the statistical errors. The value of $T_{c} / \sqrt{\sigma_{0}}$, obtained by combining the precise determination of $a^{2} \sigma_{0}$ with the deconfined temperaure $T_{c}$, is an important universal quantity which characterizes the particular gauge theory; the small variations appearing in Table 2 are presumably due to the corrections-to-scaling that we have neglected. Nonetheless, we can assert the value closer to the continuum limit is that obtained in the simulation with bond percolation and $\ell_{c}=8$ where statistical and systematic error were better under control; therefore we will use, in the following, $T_{c} / \sqrt{\sigma_{0}}=1.4878(5)$. 
If we plot the adimensional ratio $\sigma(T) / T_{c}^{2}$ versus the reduced temperature $t=\left(T-T_{c}\right) / T_{c}$ it turns out that all data lie almost on a unique curve, see Figure 3; this non-universal behaviour is related to the fact the five different systems are characterized by different "universal" value of the quantity $T_{c} / \sqrt{\sigma_{0}}$ and the adimensional variables used are very sensitive to it. As a matter of fact, if we impose the value of $\sigma(T) / T_{c}^{2}$ is the same for all systems, i.e. we determine for each system a new $T_{c}$ value by which $T_{c} / \sqrt{\sigma_{0}}=1.4878(5)$, all data fall on a unique universal curve as Figure $\$$ shows. This is the most important result of this work because it shows our results are independent of the regularisation used, therefore we are studing a "real" gauge theory well defined in the continuum limit.

It is interesting to note that the values of $C$ and of $T_{c} / \sqrt{\sigma_{0}}$ can be determined with only two pieces of information: (1) the data are all in the scaling region and (2) they show a linear behaviour in the range $-0.55<t<-0.225$. This means we can impose the two following equations to coincide in the above range $\left(S=\frac{\sigma_{0}}{T_{c}^{2}}\right.$ and $\left.x=\frac{T}{T_{c}}\right)$ :

$$
\begin{aligned}
\frac{\sigma(L)}{T_{c}^{2}} & =S-\frac{\pi}{6} x^{2}-\frac{\pi^{2}}{72 S} x^{4}+\frac{\pi^{3}}{C S^{2}} x^{6}, \\
\frac{\sigma(L)}{T_{c}^{2}} & =A(x-1) .
\end{aligned}
$$

Immediately, without using numerical data, it is possible to determine $C \simeq 290$ and $T_{c} / \sqrt{\sigma_{0}} \simeq$ 1.4884; these two values are remarkably close to those obtained using the numerical data, see Table 2. In Figure 4 we plot Eq. (4.2) (dashed line) and Eq. (4.3) (dotted line) using those values; the numerical data lie on the two curves in the scaling region. This is an important observation because it means the two quantity, $C$ and $T_{c} / \sqrt{\sigma_{0}}$ are constrained to each other in that region.

\section{Conclusions}

In this paper we have studied, by numerical simulation, the gauge theory dual to the percolation model; we can conclude it is possible to describe the long distance dynamics of this theory by means of an effective string picture. Our numerical experiment demonstrate that the quantities which characterize the effective string theory do not depend on the specific regularisation used. Moreover, we determined with high precision the value of $\sigma(T) / T_{c}^{2}$ and, for the first time in a gauge theory, we have determined the value of the $T^{6}$ coefficient $C$ of the string tension $\sigma(T)$. Furthermore, it was possible to show that the universal ratio $T_{c} / \sqrt{\sigma_{0}}$ and the coefficient $C$ are bound together in the scaling region.

\section{References}

[1] R. D. Pisarski and O. Alvarez, Phys. Rev. D 26 (1982) 3735.

[2] M. Lüscher and P. Weisz, JHEP 0407 (2004) 014 [arXiv:hep-th/0406205].

[3] J. F. Arvis, Phys. Lett. B 127 (1983) 106.

[4] K. Dietz and T. Filk, Phys. Rev. D 27 (1983) 2944.

[5] J. Polchinski and A. Strominger, Phys. Rev. Lett. 67 (1991) 1681. 


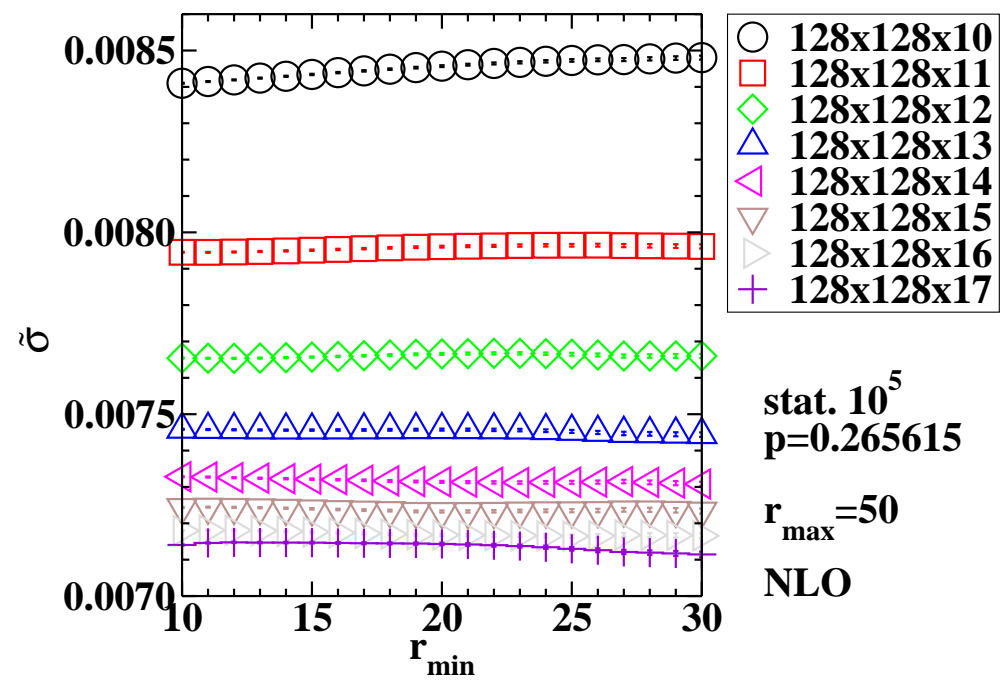

Figure 1: The fitted value of the string tension $\tilde{\sigma}$ as a function of the minimal distance $r_{\min }$ of the set of Polyakov-Polyakov correlators considered in the fit; case of bond percolation with $\ell_{c}=8$.

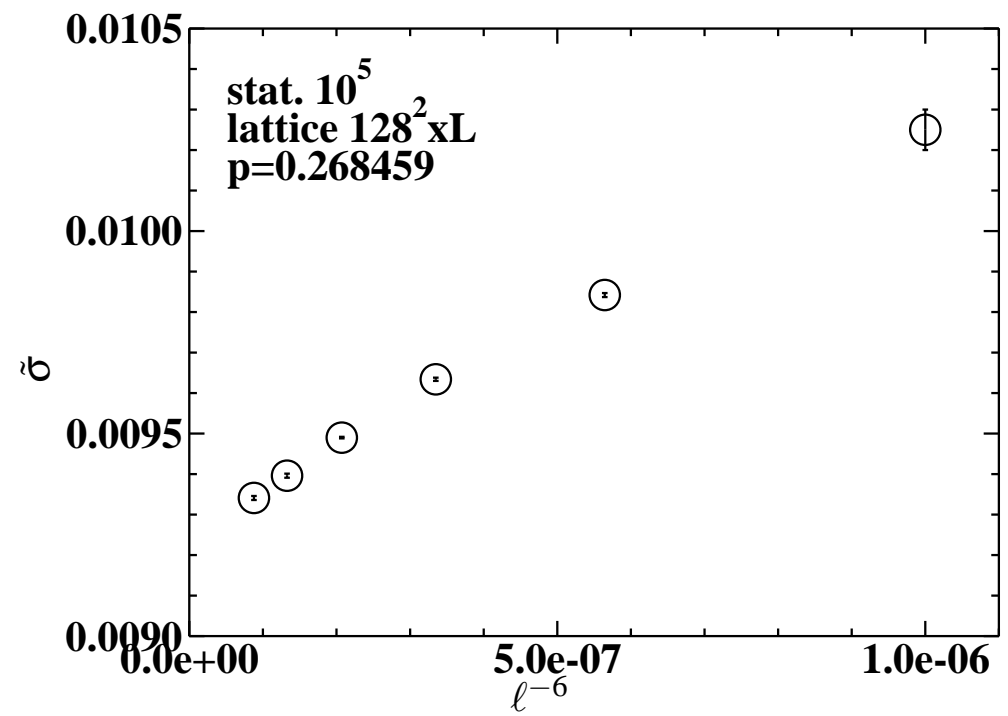

Figure 2: Plot of the fitting parameter $\tilde{\sigma}$ as a function of $T^{6}$ in numerical experiments with bond percolation with $\ell_{c}=7$.

[6] F. Gliozzi, S. Lottini, M. Panero and A. Rago, Nucl. Phys. B 719 (2005) 255 [arXiv:cond-mat/0502339].

[7] P. Giudice, F. Gliozzi and S. Lottini, in preparation.

[8] H. A. Kramers and G. H. Wannier, Phys. Rev. 60 (1941) 252.

[9] C. M. Fortuin and P. W. Kasteleyn, Physica 57 (1972) 536.

[10] In this conference the talk of Stefano Lottini, PoS LAT2008 (2008) 265.

[11] P. Giudice, F. Gliozzi and S. Lottini, PoS LAT2007 (2007) 314 [arXiv:0709.4336 [hep-lat]]. 


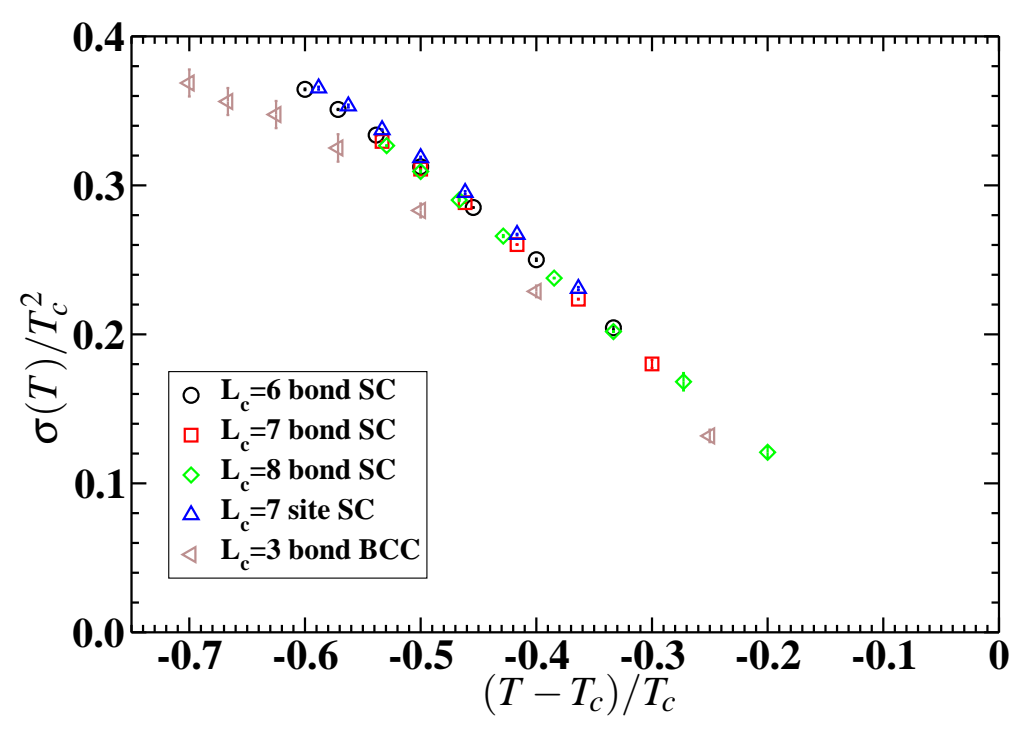

Figure 3: Plot of the scaling variable $\sigma(T) / T_{c}^{2}$ versus the reduced temperature.

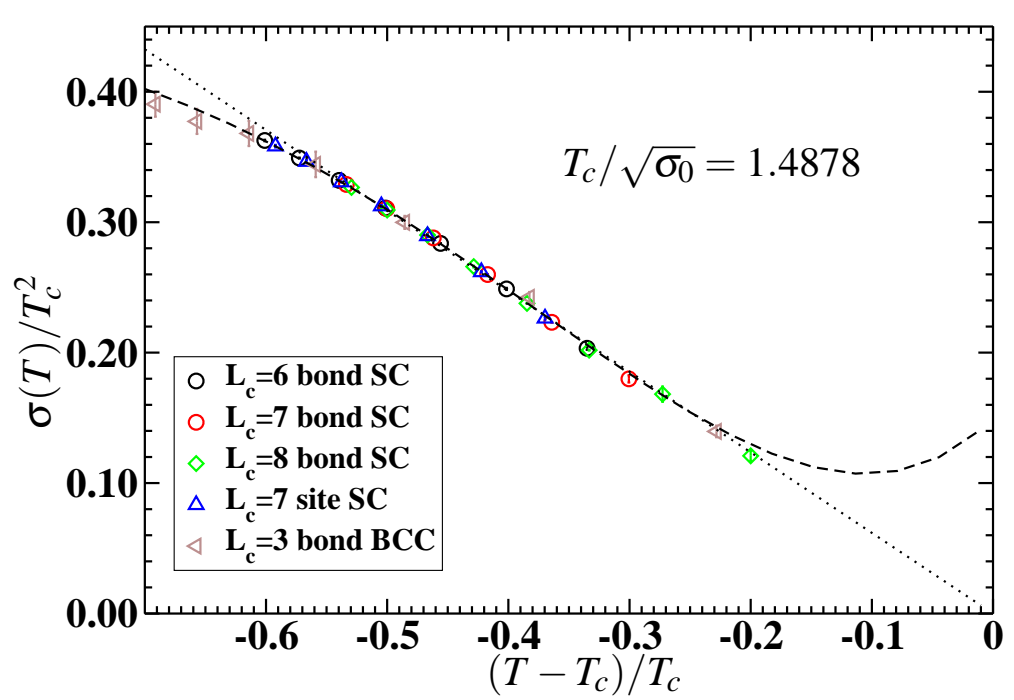

Figure 4: Plot of the scaling variable $\sigma(T) / T_{c}^{2}$ versus the reduced temperature when we impose the value of $T_{c} / \sqrt{\sigma_{0}}=1.4878$. Dashed line is the plot of Eq. (4.2), dotted line is that of Eq. (4.3). 